REVISTA

MEXICANA DE

ECONOMÍA Y

FINANZAS

REMEF

(THE MEXICAN JOLRNAL OF

ECONOMICS AND FINANCE)
Revista Mexicana de Economía y Finanzas, Nueva Época

Volumen 16 Número 3, Julio - Septiembre 2021, pp. 1-22, e654

THE ECONOMICS AND FINANCE EFFECTS OF THE COVID-19 PANDEMIC Editor: Dr. Ignacio Perrotini

DOI: https://doi.org/10.21919/remef.v16i3.654

(Recibido: 26/febrero/2021, aceptado: 29/junio/2021, publicado: 30/junio/2021)

\title{
Impacto de la pandemia COVID-19 en los precios de la gasolina y el gas natural en las principales economías de Latinoamérica
}

\author{
Ricardo Jacob Mendoza-Rivera ${ }^{1}$ - Instituto Politécnico Nacional, México \\ Francisco Venegas-Martínez ${ }^{2}$ - Instituto Politécnico Nacional, México
}

El objetivo de este trabajo es evaluar el impacto de la pandemia COVID-19, medida a través del índice de fatalidad, en los precios de la gasolina y el gas natural en las principales economías en Latinoamérica: Brasil, México, Colombia, Perú, Chile y Uruguay. Estas economías no sólo son las más grandes de la región, sino también las más afectadas por la pandemia de COVID-19. Asimismo, estos países han mostrado, en las últimas décadas, una demanda ascendente de gasolina y gas natural. Esta investigación utiliza diversos modelos de datos panel con datos semanales (febrero 2020-febrero 2021). Los modelos de datos panel de efectos aleatorios a dos vías sugieren evidencia empírica de que las tasas de crecimiento de los índices de fatalidad, para todos los países en la muestra, tienen efectos negativos únicamente en las tasas de crecimiento de los precios de gasolina; sin ningún efecto en el precio del gas. En esta investigación, el tipo de cambio es utilizado como variable de control por su relación con los precios de los hidrocarburos. Los datos empleados en el análisis son oficiales sin considerar el exceso de mortalidad por la causa específica del COVID-19. Este tipo de análisis en Latinoamérica, hasta donde los autores saben, es novedoso y contribuye a la discusión de coyuntura entre la crisis de salud y su relación con la volatilidad de los precios de los energéticos.

Clasificación JEL: Q41, F31, C23.

Palabras clave: gas natural, gasolina, tipo de cambio, modelos de datos panel, COVID-19.

\section{Impact of the COVID-19 Pandemic on Gasoline and Natural Gas Prices in the Major Latin American Economies}

This paper is aimed at evaluating the impact of the COVID-19 pandemic, measured through the fatality index, on the gasoline and natural gas prices in the main Latin American economies: Brazil, Mexico, Colombia, Peru, Chile and Uruguay. These economies are not only the largest in the region, but also the most affected by the COVID-19 pandemic. Likewise, these countries have shown, in the last decades, a growing demand for gasoline and natural gas. This research uses several panel data models with weekly data (February 2020 - February 2021). Two-way random-effects panel data models suggest empirical evidence that mortality rate growth rates, for all sample countries, have negative effects only on gasoline price growth rates; without any effect on the price of gas. In this research, the exchange rate is used as a control variable due to its relationship with hydrocarbon prices. Data used in the analysis are official without considering the mortality excess by specific cause of COVID-19. This type of analysis in Latin America, as far as the authors know, is novel and contributes to the discussion of the conjuncture between the health crisis and its relationship with volatility of energy prices.

JEL Classification: Q41, F31, C23.

Keywords: natural gas, gasoline, exchange rates, panel data models, COVID-19.

${ }^{1}$ Autor de correspondencia. Email: ricardo.mendoza.ipn@gmail.com; ORCID: https://orcid.org/0000-0003-2669-9860

2 Email: fvenegas1111@yahoo.com.mx; ORCID: https://orcid.org/0000-0003-1157-0298

*Sin fuente de financiamiento para el desarrollo de la investigación 


\section{Introducción}

La determinación de los precios de petróleo y gas varía de acuerdo con cada región. Por ejemplo, Estados Unidos y Reino Unido responden a un equilibrio competitivo con la fuerza de mercado. Por otro lado, Rusia mantiene precios bajos en comparación con la media internacional. Para el caso de Latinoamérica el referente es el Chicago Mercantil Exchange (CME). En este caso, la determinación de los precios del petróleo crudo y del gas natural considera, principalmente, los precios en dólares de estos energéticos en Estados Unidos, por lo que se puede esperar, en cierta medida, que el tipo de cambio esté relacionado con dicha determinación.

Por otro lado, desde los primeros meses de la pandemia de Coronavirus se generó una baja en la demanda de combustibles por la reducción de la actividad industrial y la disminución del transporte en todas sus formas. A la par, la guerra de precios del petróleo entre Rusia y Arabia Saudita en 2020 ha mostrado efectos persistentes en economías productoras de petróleo, incluyendo las de Latinoamérica. Así, todo lo anterior, en conjunto ha dado como resultado, primero, caídas bruscas e inesperadas y recuperaciones lentas y, posteriormente, algunas alzas de los precios del petróleo y del gas en los mercados internacionales; con sus consecuencias en los precios de los combustibles (Fondo Monetario Internacional, 2020).

De igual manera, la implementación de energías renovables significa una transformación gradual del sistema energético mundial, dejando en claro que el petróleo es un bien que en algún momento se reducirá significativamente su consumo. En particular, en Latinoamérica existen proyectos de mediano y largo plazo para desarrollar tecnologías que transformen a la industria y a los hogares para que utilicen energías más limpias que no dañen al medio ambiente, lo cual, a la larga, conducirá a una disminución en el uso de las gasolinas y el gas; en parte por la creciente producción de vehículos eléctricos y tecnologías limpias. Al respecto, Barbier y Burgess (2020) señalan que los países en vías de desarrollo son vulnerables a las consecuencias generadas por la pandemia COVID19 en los 17 Objetivos de Desarrollo Sostenible (Sustainable Development Goals). Sin embargo, es importante reconocer que varios programas se van a prolongar por no contar con un apoyo adicional de inmediato. Por esta razón la demanda de energéticos fósiles no disminuirá en el corto plazo en Latinoamérica. Además de que el consumo de energía, en cualquiera de sus fuentes, tiene un impacto positivo en el crecimiento económico, lo cual conlleva también a que el consumo de energéticos fósiles no se reducirá en el corto plazo (Aali-Bujari et al. 2017).

Asimismo, Abadie (2020) destaca que la pandemia tiene efectos en los mercados mayoristas de energía, reduciendo la demanda y los precios. Destacando, al mismo tiempo, que las políticas ecológicas responden a largo plazo y es por ello que muchas economías subdesarrolladas productoras de petróleo no pueden desprenderse de prácticas muy arraigadas (Amankwah-Amoah, 2020). En el corto y mediano plazo prevalece la producción de energías fósiles, principalmente en países en desarrollo; como el caso de Latinoamérica. En contraste, las energías renovables han retomado un papel importante en la demanda, pues los contaminantes también están en escrutinio de los tomadores de decisiones y es importante tomarlo en cuenta para minimizar lo que ahora son llamados los riesgos ecológicos (Abadie et al. 2020). 
El contexto de la pandemia, a nivel global, combina una crisis de salud con un desbalance entre la oferta y demanda agregadas. Por esta situación, para muchas economías en Latinoamérica, la salud financiera y estructural del sector petrolero no ha sido muy buena, por lo que los mercados financieros ${ }^{3}$ ya descontaron la limitada disciplina de capital en la industria petrolera, lo cual ha generado un círculo vicioso entre el pobre desempeño de la industria petrolera y los precios de los energéticos fósiles (Barbosa et al. 2020).

Por otro lado, Meher et al. (2020) analizan el impacto de la pandemia de COVID-19 en mercados de "commodities", incluyendo el petróleo y el gas. Estos autores destacan que debido a la crisis de demanda y la disminución de la oferta y las caídas en los precios del crudo, así como su lenta recuperación es preocupante para los países productores de petróleo pues su principal bien mineral es la parte más relevante de su comercio con el resto del mundo. Por último, estos autores enfatizan que una forma de medir el impacto de esta crisis es por el efecto de apalancamiento de COVID-19 con la volatilidad de los precios de los "commodities".

El principal objetivo de esta investigación consiste en examinar el impacto de la pandemia COVID-19, medida a través del índice de fatalidad (razón entre fallecimientos e infectados confirmados), en los precios de algunos de los hidrocarburos de mayor consumo, gasolina y gas natural, en las principales economías en Latinoamérica: México, Brasil, Chile, Colombia, Uruguay y Perú. Para alcanzar el objetivo de esta investigación se desarrollan varios modelos de datos panel con diferentes características, utilizando datos semanales entre febrero de 2020 y febrero de 2021. El tipo de cambio se utiliza en la presente investigación como variable de control. Es importante destacar que las economías en la muestra no sólo son las más grandes de la región, sino también las más afectadas por la pandemia de COVID-19. Asimismo, estos países han mostrado una demanda ascendente de petróleo y gas natural en las últimas décadas.

La presente investigación se distingue con respecto al estado actual del tema en lo siguiente: 1) se concentra en la economías más grandes de Latinoamérica y con la mayor letalidad de COVID19, 2) se cuenta con una mayor disponibilidad de datos de precios de petróleo y gas natural, así como datos de COVID-19 con respecto del pasado, 3) se estiman diversos modelos de datos panel que permiten utilizar una mayor cantidad de países, variables y períodos; y 4) se contribuye a la discusión de coyuntura entre la crisis de salud y su relación con las volatilidad de los precios de los energéticos.

Este trabajo está organizado de la siguiente manera: la sección 2 hace una breve discusión de cómo medir los efectos de la pandemia por COVID-19 en los mercados de energéticos; la sección 3 examina el comportamiento de los datos de la muestra de los países seleccionados; la sección 4 identifica la especificación econométrica más adecuada; la sección 5 realiza una discusión de los resultados empíricos obtenidos; por último, la sección 6 proporciona las conclusiones.

\section{Estado del arte}

Bolton et al. (2020) destaca que los modelos predictivos desarrollados antes de la pandemia no estaban diseñados para tratar con crisis de incertidumbre en la salud, tal como lo había sugerido anteriormente Stiglitz (2017). Por otro lado, Barbosa et al. (2020) sugieren que deben realizarse

\footnotetext{
${ }^{3} \mathrm{Al}$ respecto, una investigación que examina los efectos de la pandemia de COVID-19 en diversas variables financieras en Latinoamérica se encuentra en Mendoza-Rivera et al. (2020).
} 
cambios fundamentales en las visiones que han prevalecido por décadas e incorporar nuevos supuestos.

La gran mayoría de los modelos "disponibles" no incluyen choques en la salud como una variable clave. Los efectos en la demanda agregada que se han visto como producto de la incertidumbre generada por el COVID-19 han sido más que rebasados por cualquier pronóstico elaborado con modelos con supuestos estándar; tal vez por no incluir, en general, a la salud. En conclusión, ningún modelo pudo predecir el rumbo de la actual crisis global COVID-19.

Por otro lado, Summers (2000) enfatiza que un agente que jugará un papel fundamental en la intermediación de los mercados durante la crisis es la banca internacional que debe ser incluida como un sector con mayor relevancia en los modelos tradicionales. También, es importante mencionar que el gerente general del BIS, Agustín Carstens, argumenta la importancia de contar con una política monetaria adaptada a esta crisis COVID-19. Asimismo, El Banco Mundial junto con el Fondo Monetario Internacional manifestaron su compromiso de brindar apoyo a los países miembros para hacer frente al impacto sanitario y económico del COVID-19 (Clavellina-Miller y Domínguez-Rivas, 2020).

A un año de la pandemia por COVID-19 existen estudios empíricos que relacionan sus efectos sobre los precios de los energéticos y otras variables. Por ejemplo, Gómez et al. (2020) analizan la causalidad predictiva entre los tipos de cambio y los precios del petróleo y encuentran que en el caso de países productores, los precios del petróleo son receptores del tipo de cambio, mientras que en los países importadores muestran que la relación es más fuerte de los precios del petróleo al tipo de cambio. Por su parte Villarreal-Samaniego (2021) estudia la relación entre el precio del petróleo y el tipo de cambio en el primer trimestre de la pandemia en diversas economías en donde se destaca que sí existen relaciones causales.

Los estudios que revelan efectos entre los precios de energéticos y el tipo de cambio han sido estudiados inclusive antes de la pandemia, esto por los efectos en las negociaciones internacionales, exportaciones e importaciones (López-Herrera y Rodríguez-Benavides, 2020; Carvajal y Bermúdez, 2020; Kim y Jung, 2018; y Thenmozhi y Srinivasan, 2016).

En lo que corresponde al gas natural, los precios también has sufrido efectos por la pandemia, y aunque en menor medida que el petróleo, la demanda en el primer semestre de 2020 por la falta de actividad económica agudizó la competencia entre los suministradores tradicionales, siendo los EEUU uno más afectados, que no sólo distribuye en América, sino también tiene una fuerte posición en Europa.

Los efectos de los factores económicos, políticos y de salubridad han cambiado las cotizaciones en el sector energético y también las primas de riesgo de los países productores, incrementando la volatilidad de los precios y el crecimiento en el riesgo (Escribano y Lázaro, 2020). A pesar de que la incertidumbre provocada por la crisis sanitaria se encuentra en mayor control, el choque de demanda persiste en los mercados de petróleo y gas natural. 


\section{Naturaleza de los datos}

El criterio para elegir la muestra de países latinoamericanos para la presente investigación no sólo toma en cuenta el tamaño de la economía, sino también los efectos más severos por la pandemia de COVID-19. Los países seleccionados son: México, Brasil, Chile, Colombia, Uruguay y Perú. A continuación se analiza el comportamiento de las variables bajo estudio.

\section{1 Índice de fatalidad por COVID-19}

La tasa, o índice, de fatalidad (fatality ratio) es la proporción de individuos que fallecen por COVID19 con respecto de los casos afectados (confirmados positivo) en un tiempo dado, $t$. De esta manera la tasa de fatalidad, $I F_{t}$, está dado por:

$$
I F_{t}=\frac{M_{t}}{A_{t}} \times 100
$$

donde $M_{t}$ es el número de fallecimientos por COVID-19 y $A_{t}$ son los casos afectados por COVID-19 en la semana $t$.

Las dinámicas de los índices de fatalidad en los diferentes países de la muestra difieren en días al inicio de casos confirmados. El índice de fatalidad es más general que la tasa de contagio, o tasa de infectados, ya que además de ésta el índice de fatalidad considera también el número de fallecimientos, con lo cual se refleja de manera más precisa la eficiencia de las políticas públicas de salubridad contra la pandemia en cada país de la muestra. La Gráfica 1 muestra los cambios en el índice de letalidad desde el inicio de los primeros casos confirmados, es decir, desde el 26 de febrero de 2020 y considera observaciones semanales hasta el 28 de febrero de 2021. En la Gráfica 1 se destaca que la tasa máxima de fatalidad difiere en tiempo para los diferentes países, para Brasil y Colombia el efecto máximo se tuvo entre las semanas 9 y 13, es decir, entre abril y mayo de 2020. Por otro lado Uruguay tuvo un efecto más estable con una curva más plana y un desarrollo más lento de la pandemia, teniendo el mayor efecto entre las semanas 22 y 24, es decir, entre julio y agosto 2020. En Chile y Perú se observa un efecto ascendente con ligeras bajas a lo largo del año. En particular, en Perú se observa su máxima fatalidad entre las semanas 23 y 29, es decir, entre julio y septiembre 2020. México representa el país con la mayor tasa de fatalidad desde el inicio de la pandemia y casi duplica la tasa de los países de la muestra desde la semana 10, es decir, a finales de abril 2020. Además su máximo se alcanza en la semana 20, a principios de julio de 2020. 


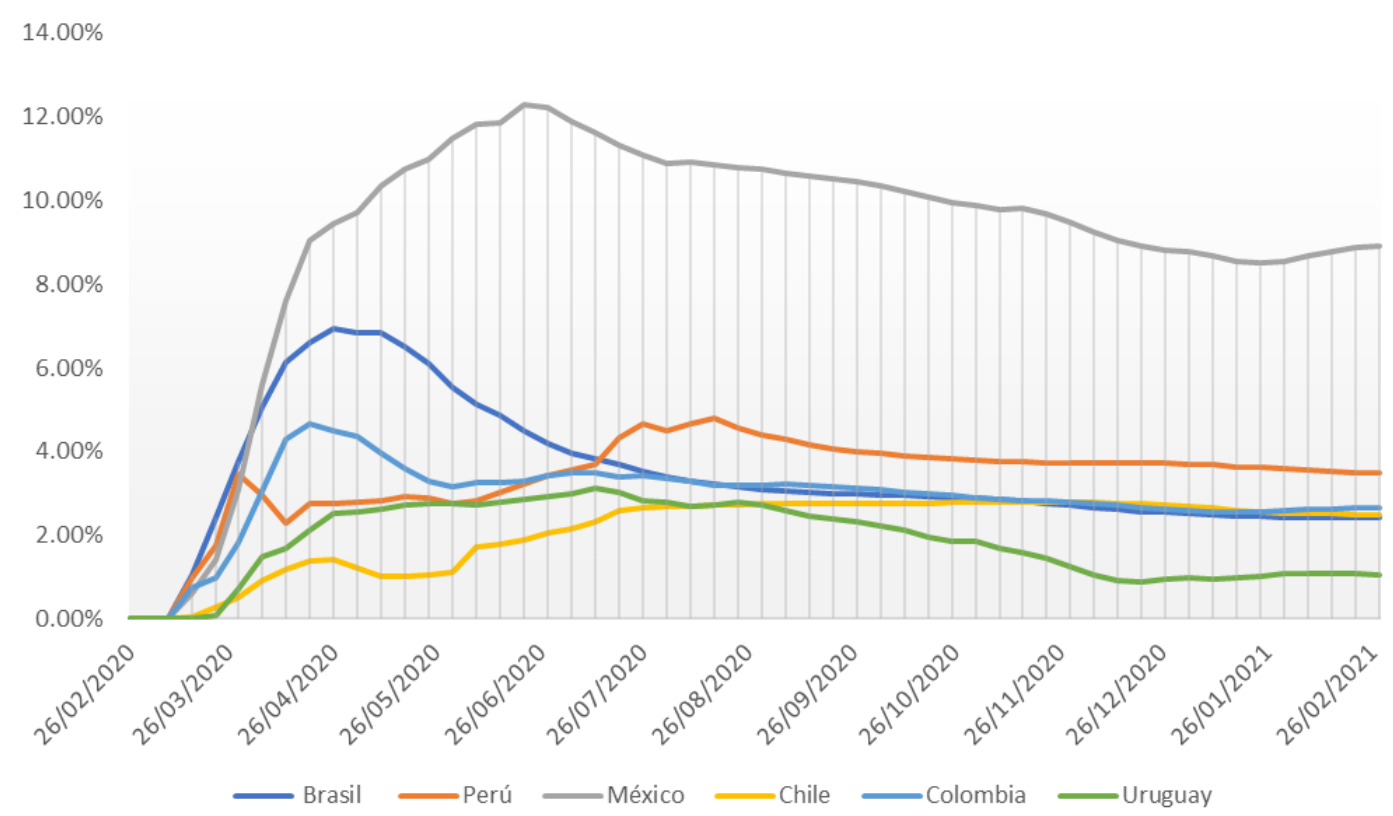

Gráfica 1. Índice de fatalidad de la muestra en América Latina

Fuente: Elaboración propia con datos en.wikipedia.org/wiki/COVID-19_pandemic

Nota. La muestra comprende 54 semanas iniciando el 26 de febrero de 2020 y terminando el 28 de febrero de 2021.

\subsection{Tipo de cambio USD/Moneda local}

El tipo de cambio en esta investigación se utiliza como variable de control por la relación que guarda con los precios de los hidrocarburos en todo el mundo. El tipo de cambio con el dólar americano (USD) es una variable fundamental en cualquier economía por las transacciones y los precios futuros pactados para realizar múltiples operaciones con el resto del mundo, y porque diversos indicadores macroeconómicos se referencian en USD (PIB per cápita, tasa de interés real, competitividad, etc.). Las monedas locales de la muestra de países latinoamericanos son: en Brasil el real brasileño, BRL; en Perú el sol peruano, PEN; en México el peso mexicano, MXN; en Chile el peso chileno, CLP; en Colombia el peso colombiano, COP; y, por último, para Uruguay el peso uruguayo, UYU. En la Gráfica 2 se muestran los tipos de cambio de las monedas locales contra el dólar americano de la muestra de países estudiados. En esta gráfica se observa un proceso de volatilidad desde el inicio del confinamiento de la pandemia para todos los países y hasta febrero de 2021. A partir de agosto de 2020 se observa una mayor estabilidad en los tipos de cambio e incluso con tendencias decrecientes como el caso de Brasil y México. 


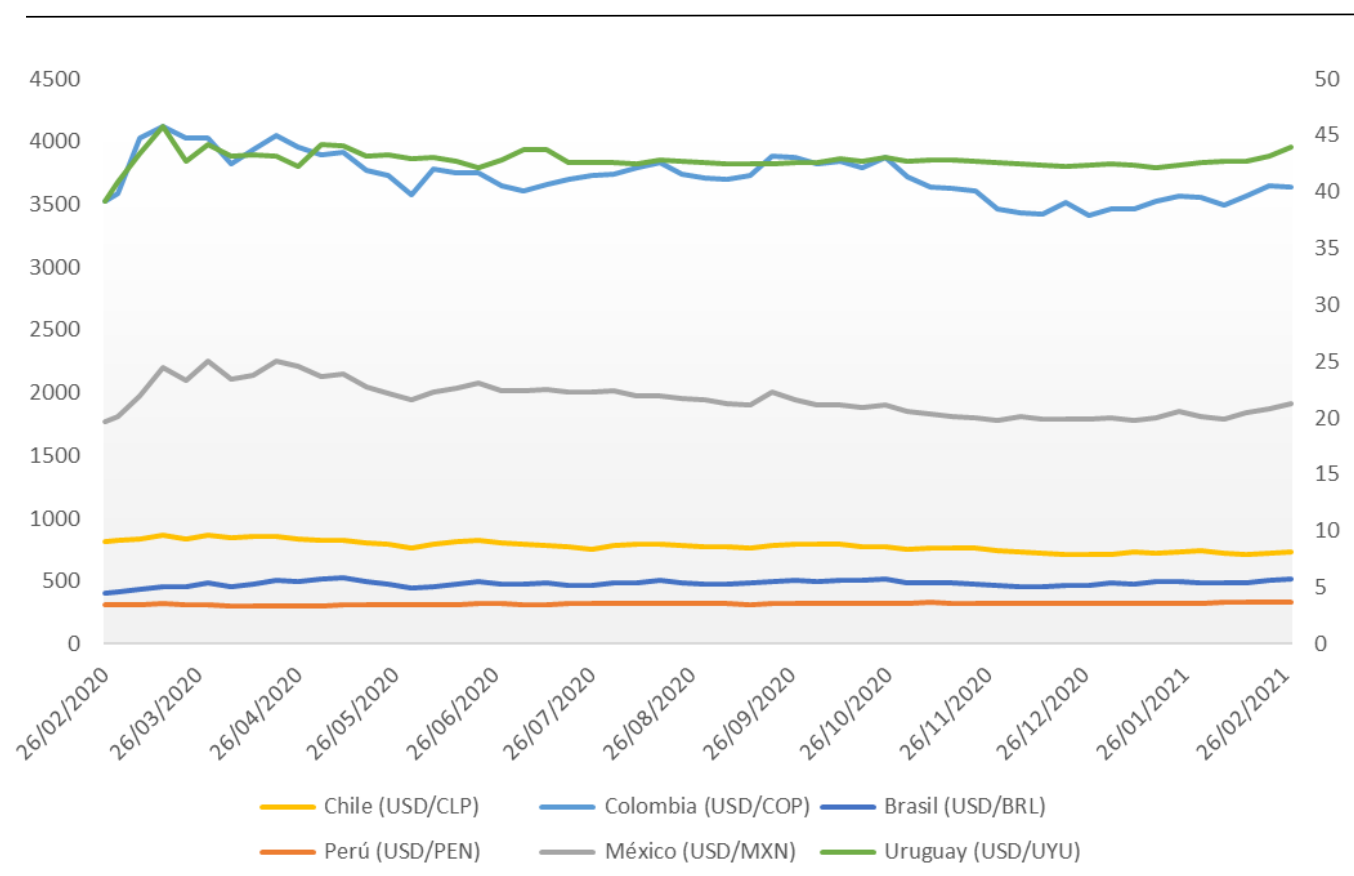

Gráfica 2. Tipos de cambio Moneda local-USD

Fuente: Elaboración propia con datos de Investing.com

Con respecto de las tasas de depreciación del tipo de cambio (tasas de crecimiento del tipo de cambio), la Gráfica 3 muestra efectos muy acentuados en la dinámica de las tasas de depreciación en el primer trimestre de la pandemia. Las tendencias se hicieron más estables conforme avanzaba el tiempo. También se puede observar que los efectos en general son mayormente visibles hacia arriba.

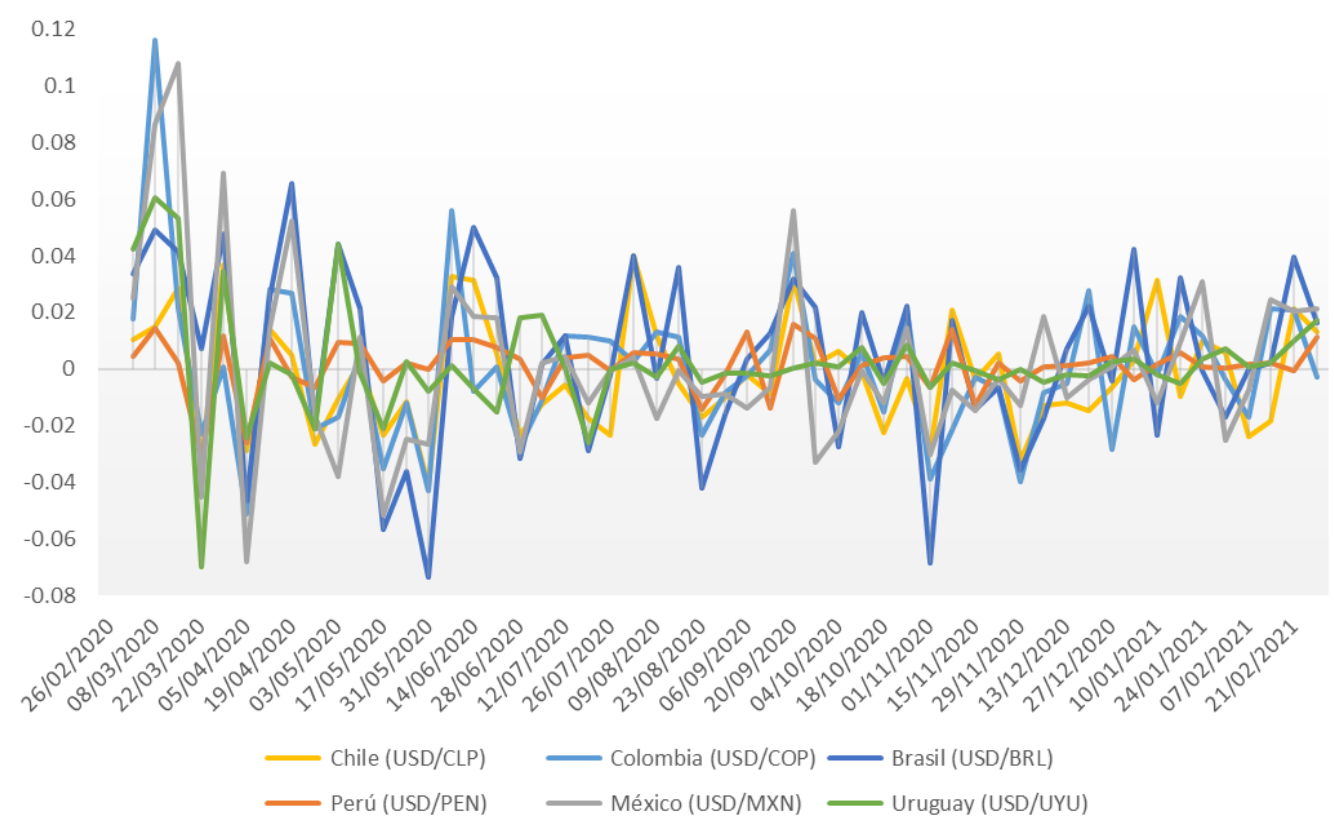

Gráfica 3. Tasas de crecimiento de los tipos de cambio Moneda local-USD

Fuente: Elaboración propia con datos de Investing.com

Nota. La muestra comprende 54 semanas iniciando el 26 de febrero de 2020 y terminando el 28 de febrero de 2021. 
Por último, es importante mencionar que Ramelli y Wagner (2020) argumentan que el mercado responde a la incertidumbre sobre las consecuencias económicas del coronavirus. Esto se detectó principalmente en el comercio internacional, donde los precios del mercado agregado y los inversionistas se vieron afectados en los canales financieros ante el avance de la pandemia COVID19. Algunos de los canales más susceptibles fueron el mercado cambiario y el bursátil.

\subsection{Precios de la gasolina}

El consumo de gasolinas es importante en los hogares y en el quehacer industrial al transportar insumos y bienes, representado un factor trascendental en la actividad económica. Existen estudios que sugieren que el consumo de gasolinas se verá incrementado en los próximos años a pesar de la producción acrecentada de automóviles híbridos y eléctricos. En Latinoamérica la gasolina, y otros combustibles, influyen de manera definitiva en el crecimiento económico (Galindo y Reyes, 2015).

El inicio de la pandemia por COVID-19 trajo consigo una baja en el consumo de energéticos debido al proceso de confinamiento que se ha implementado en la mayoría de los países. La industria, en general, ha disminuido su producción y las familias no recorren en sus vehículos las distancias que en la normalidad acostumbraban. Estos efectos son visibles en la Gráfica 4 donde se observa un deterioro en el precio durante la época más fuerte del confinamiento. Se observa además otro deterioro al finalizar el año 2020 cuando la mayoría de los países comenzaron a sufrir un rebrote de casos y se determinaban medidas más restrictivas. La recuperación en 2021 es notable en la mayoría de las economías hasta la fecha final de este estudio.

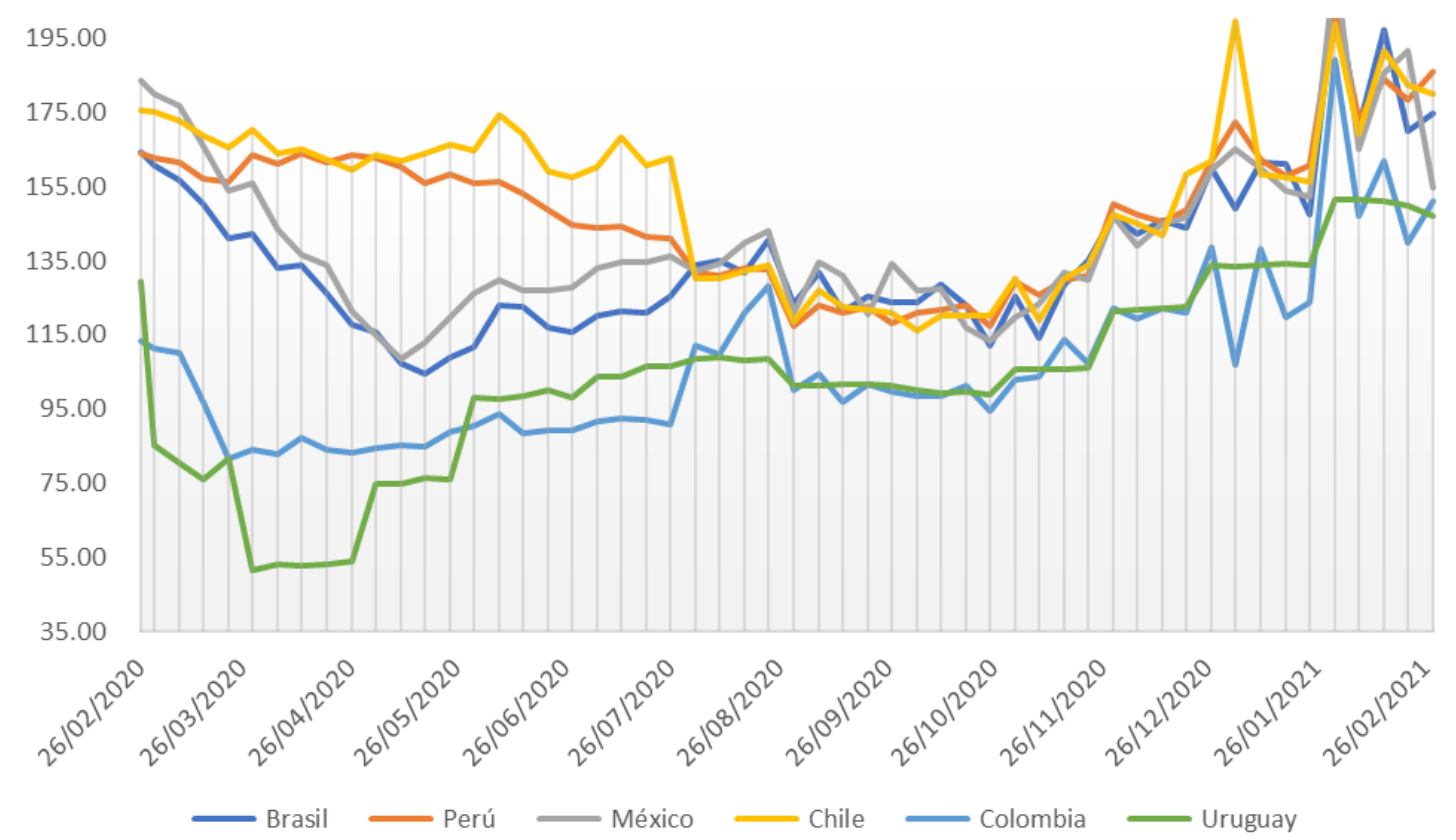

Gráfica 4. Precios de la gasolina (USD/Barril)

Fuente: Elaboración propia con datos de Globalpetrolprices.com \& indexmundi.com 
Con respecto de las tasas de crecimiento de los precios de la gasolina (USD/Barril) se observa un deterioro muy marcado en el primer bimestre de la pandemia, cuando las economías de estudio declararon su máximo confinamiento. Posteriormente se observa una ligera recuperación en todos los países, a excepción de Uruguay. Las fluctuaciones comienzan en precios muy bajos y se mueven hacia una recuperación más sostenida como se aprecia en la Gráfica 5.

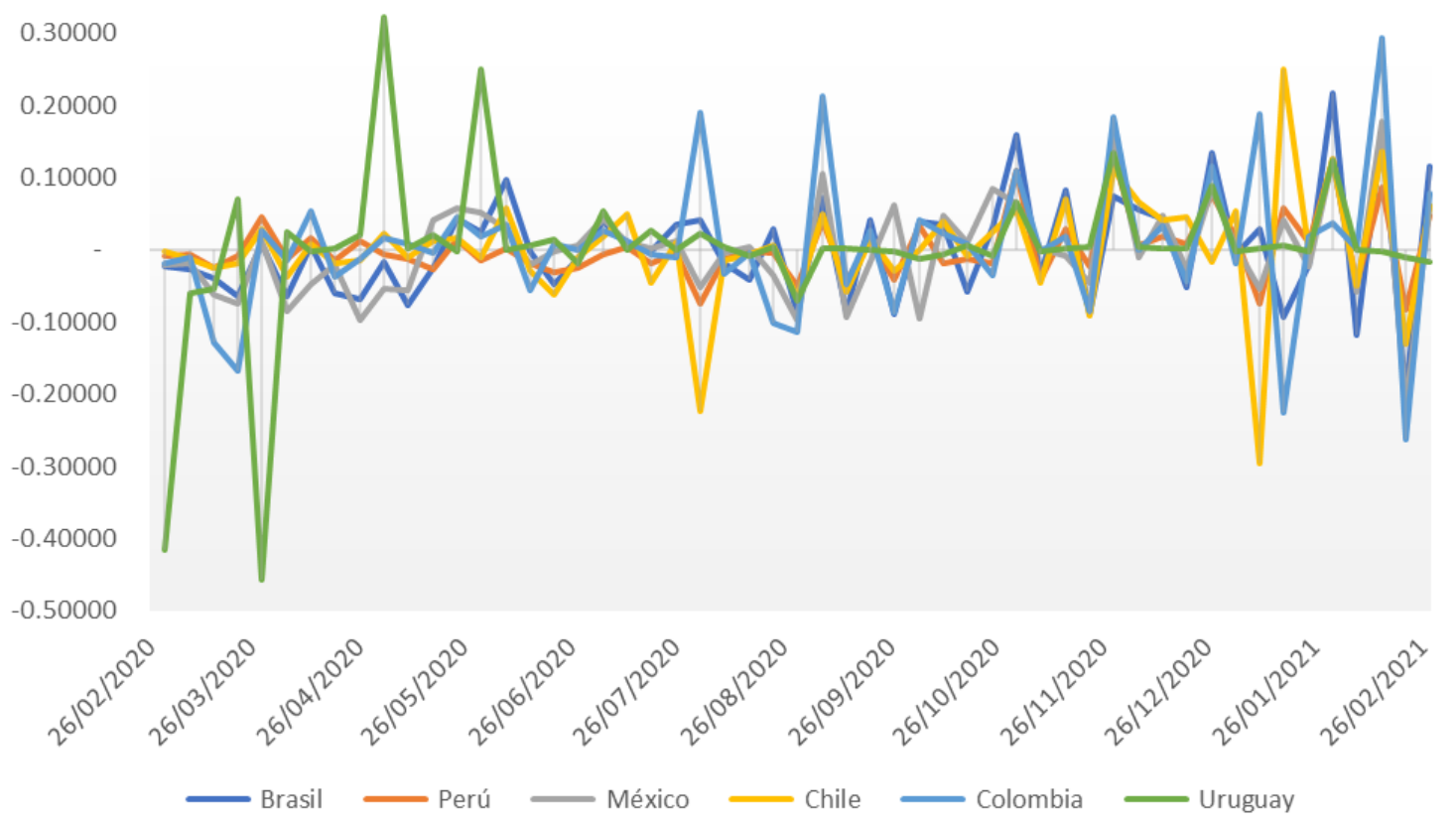

Gráfica 5. Tasas de crecimiento de los precios de la gasolina (USD/Barril)

Fuente: Elaboración propia con datos de Globalpetrolprices.com \& indexmundi.com

Nota. La muestra comprende 54 semanas iniciando el 26 de febrero de 2020 y terminando el 28 de febrero de 2021.

\subsection{Precios de gas natural}

El mercado de gas natural a nivel mundial se puede dividir en zonas geográficas por distribución de las reservas de gas natural en el mundo como se aprecia en la Tabla 1.4

Tabla 1. Distribución de reservas de gas natural mundial

\begin{tabular}{|c|c|c|}
\hline Zona geográfica & Trillones $m^{3}$ & $\mathbf{\%}$ \\
\hline Norte América & 13.9 & 7.1 \\
\hline Sur y Centro América & 8.2 & 4.2 \\
\hline Europa & 3.9 & 2.0 \\
\hline
\end{tabular}

${ }^{4}$ El gas se origina como consecuencia de la descomposición durante miles de años de materia orgánica enterrada con otros sedimentos a altas presiones y altas temperaturas en el interior de yacimientos a grandes profundidades, de donde es extraído, por lo cual es considerado un combustible fósil. 


\begin{tabular}{|c|c|c|}
\hline Comunidad de Estados Independientes & 62.8 & 31.9 \\
\hline Medio Oriente & 75.5 & 38.4 \\
\hline África & 14.4 & 7.3 \\
\hline Asia pacífico & 18.1 & 9.2 \\
\hline Total & $\mathbf{1 9 6 . 9}$ & $\mathbf{1 0 0}$ \\
\hline
\end{tabular}

Fuente: Elaboración propia con datos de British Petroleum (BP) (2019)

Nota. Datos al cierre de 2018

El gas natural se ha convertido en el principal combustible para la generación de energía eléctrica y, en menor medida. en la producción y el transporte de bienes. El precio del gas se fija utilizando referencias en precios de Estados Unidos, es por esto que la dirección que toman los precios son un tanto estáticos y no fluyen con las fuerzas de mercados locales, a diferencia del caso de la gasolina.

En la Gráfica 6 se observa un comportamiento similar de los precios del gas natural en la mayoría de las economías de la muestra, valuados sobre el precio spot Henry Hub. En dicha Gráfica se observa que los precios fueron más estables en el periodo de mayor confinamiento. Sin embargo, en julio y octubre de observan caídas abruptas en el precio, excepto para Uruguay, con una recuperación más rápida y sostenida.

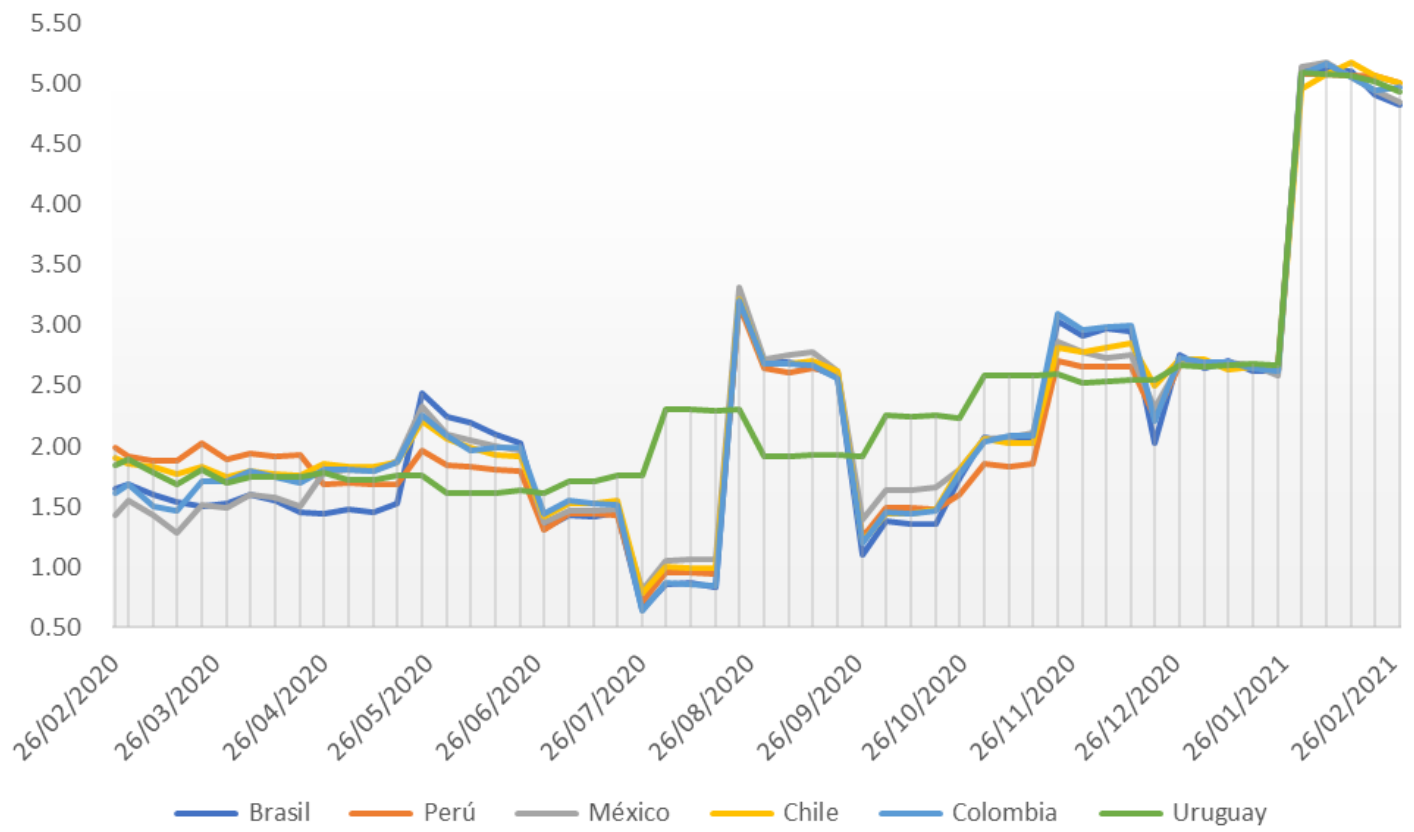

Gráfica 6. Precios de gas natural (USD/MBtu)

Fuente: Elaboración propia con datos de indexmundi.com

En cuanto a las tasas de crecimientos de los precios de gas natural se observan, en la Gráfica 7, dinámicas similares a través del tiempo en todas las economías bajo estudio. Los mayores efectos se observan entre junio de 2020 y octubre de 2020. Esto se puede interpretar como un efecto retardado del confinamiento y la relación con los precios del petróleo. 


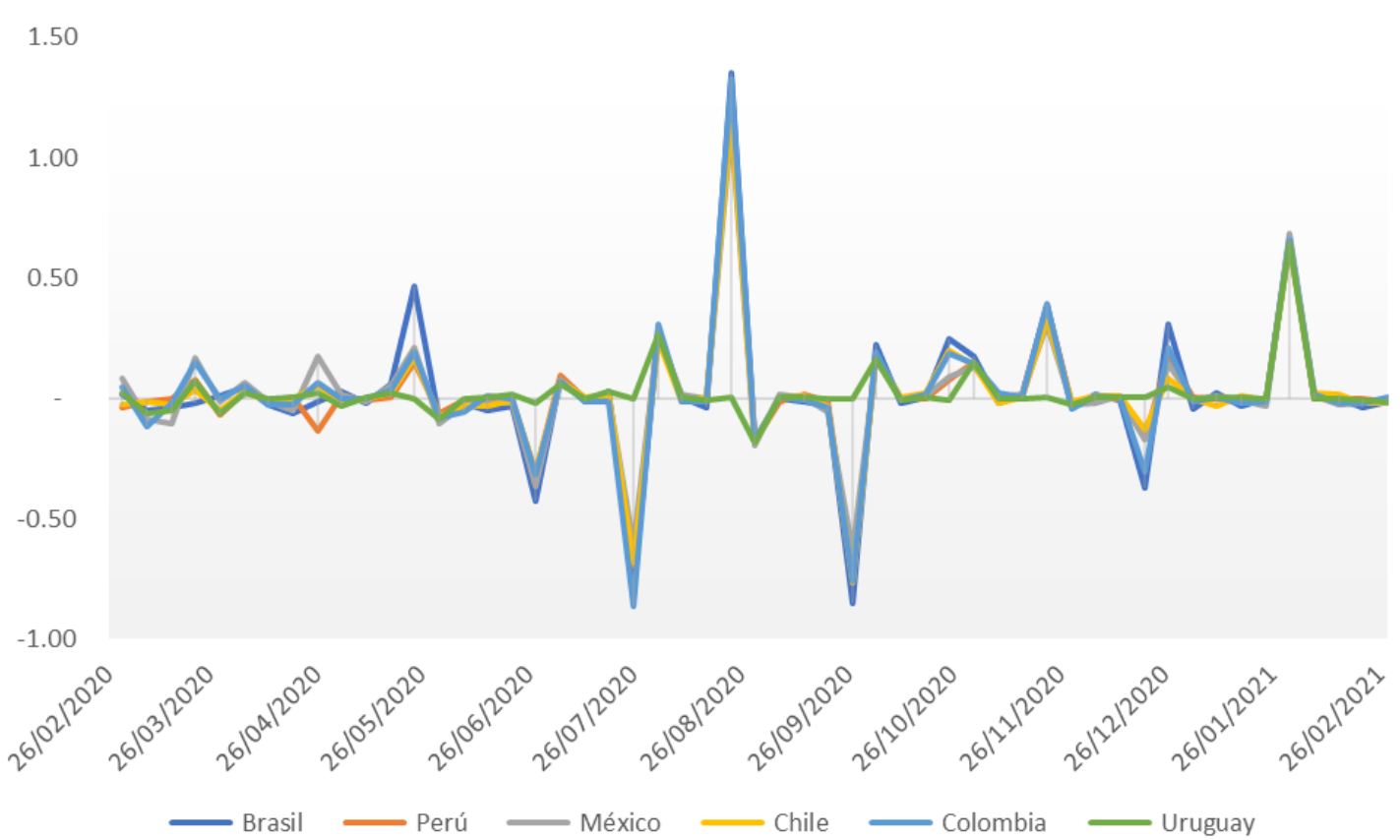

Gráfica 7. Tasas de crecimiento de los precios de gas natural (USD/MBtu)

Fuente: Elaboración propia con datos de indexmundi.com

Nota. La muestra comprende 54 semanas iniciando el 26 de febrero de 2020 y terminando el 28 de febrero de 2021.

\section{Metodología y especificación econométrica}

Cuando se considera información perteneciente a diferentes países (unidades), es decir, cuando se tiene un corte transversal y, además, esta información contiene series temporales entonces una especificación econométrica adecuada podría ser la de un modelo de datos panel (Arellano y Bover, 1990). Existen, por supuesto, otros modelos econométricos que analizan información de series temporales. Sin embargo, por la naturaleza de los datos y al considerar un año de la pandemia, es más adecuado emplear el modelo de datos panel.

El modelo de datos panel que se emplea en esta investigación considera un conjunto de países y además información disponible semanalmente de variables de dichos países. Los subíndices de los países son $i=1,2, \ldots, N=6$, las observaciones de corte transversal, y los subíndices de corte temporal se denotan por $t=1,2, \ldots, T=54$. Así pues, la especificación de datos panel se muestra en la siguiente ecuación:

$$
y_{i t}=\alpha_{i t}+x_{i t}{ }^{\prime} \beta+u_{i t}
$$

donde:

$y_{i t}$ : variables dependientes en un arreglo que contiene $N T \times 1$ entradas predeterminadas,

$\alpha_{i t}$ : interceptos que recolectan la heterogeneidad provocada por el efecto individual y/o temporal de las variables no observadas,

$x_{i t}$ : es la matriz de $k$ variables independientes que contiene $N T \times k$ entradas, 
$\beta$ : es el vector de parámetros de $k$ entradas,

$u_{i t} \sim N\left(0, \sigma_{u}^{2}\right)$ : es el término de error.

El modelo anterior permite capturar la heterogeneidad no observable entre variables individuales o temporales. Sin embargo, dependiendo de la la naturaleza de los datos se pueden presentar distorsiones por los efectos conjuntos de las series de tiempo. Esta investigación emplea dos modelos que difieren sobre los supuestos del intercepto y del error, los cuales proporcionan dos formas de analizar la dinámica de las variables de los países bajo estudio.

El primer modelo es el de datos panel de efectos fijos que reconoce los atributos de corte transversal y considera que existe un término constante diferente para cada unidad. Esta especificación considera que las variables explicativas afectan de la misma forma a cada unidad de estudio y estas unidades se diferencian por una característica particular medida a través del intercepto. El modelo se calcula mediante mínimos cuadrados ordinarios (MCO) y está dado por

$$
y_{i t}=\alpha_{i}+x_{i t}{ }^{\prime} \beta+u_{i t}
$$

donde:

$\alpha_{i t}$ : es una variable dicotómica que captura el efecto individual fijo.

Una limitación del modelo de datos panel de efectos fijos es la pérdida de grados de libertad cuando el número de paneles es muy grande. Además, si $N$ es muy grande, el proceso se vuelve inservible considerando un nivel de reconocimiento individual poco viable. Una alternativa que enmienda la limitación anterior es el modelo de datos panel con efectos aleatorios. Este segundo modelo supone que la ordenada al origen de cada corte transversal se extrae de forma aleatoria de una unidad con valor constante y que tiene una desviación $\varepsilon_{i}$ con respecto de su valor medio. En este caso, la estimación de los parámetros se realiza por el método generalizado de mínimos cuadrados (MGMC). En este caso se supone que el intercepto es una variable aleatoria que se puede descomponer de la siguiente forma:

$$
\alpha_{i}=\alpha+\varepsilon_{i} \quad \text { con } \quad \varepsilon_{i} \sim N\left(0, \sigma_{\varepsilon}^{2}\right) .
$$

La descripción del modelo es ahora:

$$
Y_{i t}=\alpha+x_{i t}{ }^{\prime} \beta+\varepsilon_{i}+u_{i t}
$$

Reescribiendo el término de perturbación $\omega_{i t}=\varepsilon_{i}+u_{i t}$, la ecuación anterior se transforma en:

$$
Y_{i t}=\alpha+x_{i t}^{\prime} \beta+\omega_{i t}
$$

Una limitación del modelo anterior es que $\varepsilon_{i}$ no es observable directamente, además de que se requieren supuestos sobre el término de perturbación. Al considerar los datos semanales de las 
variables bajo estudio se pretende especificar un modelo que considere los efectos de corte transversal de la muestra de países que comparten patrones del comportamiento de precios de algunos energéticos ante la pandemia y cuya repercusión a nivel temporal todavía es incierta.

Existen múltiples posibilidades para analizar el comportamiento de la ecuación (2). Las siguientes pruebas buscan identificar el modelo de datos panel que mejor explique el comportamiento de los precios de los combustibles incorporando siempre al índice de letalidad como variable independiente y al tipo de cambio como variable de control. La siguiente tabla muestra las estadísticas descriptivas de las series de tiempo para cada país donde se observan características conjuntas longitudinales tales como tendencias centrales, medidas de dispersión y curtosis.

Tabla 2. Estadística descriptiva de las variables.

\begin{tabular}{|c|c|c|c|c|c|c|c|}
\hline & FATALIDAD & GASOLINA & GAS & USD & TC_GASOLINA & TC_GAS & TC_USD \\
\hline \multicolumn{8}{|c|}{ Brasil } \\
\hline Media & 0.0341 & 136.5964 & 2.2056 & 5.35 & 0.0012 & 0.0202 & 0.0045 \\
\hline Mediana & 0.0298 & 131.7679 & 2.0280 & 5.37 & 0.0021 & 0.0111 & 0.0072 \\
\hline Máximo & 0.0692 & 207.1438 & 5.1138 & 5.86 & 0.3398 & 1.3511 & 0.0654 \\
\hline Mínimo & 0 & 104.5512 & 0.6555 & 4.47 & 0.1937 & 0.8509 & 0.0734 \\
\hline Desv. Estándar & 0.0162 & 21.7120 & 1.0953 & 0.26 & 0.0833 & 0.2975 & 0.0317 \\
\hline Kurtosis & 0.4748 & 1.3863 & 1.6271 & 2.01 & 4.1240 & 8.7095 & 0.2825 \\
\hline \multicolumn{8}{|c|}{ Perú } \\
\hline Media & 0.0335 & 148.8948 & 2.2332 & 3.54 & 0.0024 & 0.0175 & 0.0012 \\
\hline Mediana & 0.0366 & 151.7426 & 1.9010 & 3.56 & 0.0059 & 0.0023 & 0.0020 \\
\hline Máximo & 0.0480 & 203.8800 & 5.0788 & 3.69 & 0.2372 & 1.2078 & 0.0157 \\
\hline Mínimo & 0 & 117.1826 & 0.7163 & 3.37 & 0.1647 & 0.7407 & 0.0318 \\
\hline Desv. Estándar & 0.0107 & 19.5362 & 1.0484 & 0.08 & 0.0567 & 0.2547 & 0.0093 \\
\hline Kurtosis & 3.5993 & 0.1912 & 2.4950 & 0.78 & 6.0536 & 10.6021 & 2.7729 \\
\hline \multicolumn{8}{|c|}{ México } \\
\hline Media & 0.0896 & 141.5395 & 2.2458 & 21.64 & 0.0032 & 0.0229 & 0.0016 \\
\hline Mediana & 0.0980 & 134.5553 & 1.9878 & 21.58 & 0.0024 & 0.0008 & 0.0035 \\
\hline Máximo & 0.1229 & 214.4735 & 5.1716 & 25.01 & 0.3428 & 1.1290 & 0.1078 \\
\hline Mínimo & 0 & 108.6162 & 0.8050 & 19.62 & 0.2614 & 0.6358 & 0.0679 \\
\hline Desv. Estándar & 0.0314 & 22.0545 & 1.0537 & 1.47 & 0.0874 & 0.2418 & 0.0317 \\
\hline Kurtosis & 2.9980 & 1.2187 & 2.1203 & 0.50 & 4.8648 & 9.4468 & 2.2113 \\
\hline \multicolumn{8}{|c|}{ Chile } \\
\hline Media & 0.0207 & 153.4103 & 2.2833 & 781.31 & 0.0005 & 0.0183 & 0.0021 \\
\hline Mediana & 0.0257 & 159.8664 & 1.9240 & 783.39 & 0.0054 & 0.0011 & 0.0037 \\
\hline Máximo & 0.0279 & 199.5735 & 5.1712 & 866.13 & 0.2395 & 1.1730 & 0.0402 \\
\hline Mínimo & 0 & 116.1141 & 0.7880 & 708.40 & 0.2313 & 0.7703 & - $\quad 0.0412$ \\
\hline Desv. Estándar & 0.0090 & 21.9667 & 1.0389 & 42.73 & 0.0796 & 0.2487 & 0.0198 \\
\hline Kurtosis & 0.0534 & 0.8194 & 2.3297 & 0.83 & 3.0666 & 10.8016 & 0.5808 \\
\hline
\end{tabular}


REMEF (The Mexican Journal of Economics and Finance) Impacto de la pandemia COVID-19 en los precios de la gasolina y el gas natural en las principales economías de Latinoamérica

\begin{tabular}{|c|c|c|c|c|c|c|c|c|}
\hline \multicolumn{9}{|c|}{ Colombia } \\
\hline Media & 0.0287 & 107.2362 & 2.2568 & $3,716.19$ & & 0.0054 & 0.0212 & 0.0006 \\
\hline Mediana & 0.0304 & 101.6600 & 1.9761 & $3,724.82$ & & 0.0058 & 0.0008 & 0.0026 \\
\hline Máximo & 0.0468 & 188.9395 & 5.1569 & $4,118.00$ & & 0.4241 & 1.3277 & 0.1161 \\
\hline Mínimo & 0 & 81.7875 & 0.6384 & $3,414.50$ & - & 0.2606 & 0.8627 & 0.0514 \\
\hline Desv. Estándar & 0.0096 & 22.1479 & 1.0702 & 175.69 & & 0.1135 & 0.2821 & 0.0268 \\
\hline Kurtosis & 3.1135 & 2.4670 & 1.8801 & 0.59 & & 3.4714 & 10.3321 & 5.4009 \\
\hline \multicolumn{9}{|c|}{ Uruguay } \\
\hline Media & 0.0181 & 103.7484 & 2.3429 & 42.79 & & 0.0024 & 0.0186 & 0.0021 \\
\hline Mediana & 0.0191 & 102.6441 & 1.9229 & 42.66 & & 0.0016 & 0.0014 & 0.0002 \\
\hline Máximo & 0.0312 & 151.5398 & 5.0831 & 45.81 & & 0.3224 & 0.6444 & 0.0606 \\
\hline Mínimo & 0 & 51.7155 & 1.6057 & 39.18 & - & 0.4572 & 0.1830 & 0.0697 \\
\hline Desv. Estándar & 0.0093 & 25.7794 & 0.9335 & 0.85 & & 0.1079 & 0.1051 & 0.0195 \\
\hline Kurtosis & 1.0072 & - $\quad 0.1578$ & 4.0067 & 7.65 & & 10.6196 & 24.0647 & 4.6000 \\
\hline
\end{tabular}

Fuente: Elaboración propia

En la Gráfica 8 se presentan tasas de crecimiento del índice de fatalidad, del precio de la gasolina y del precio del gas y se observa que siguen una tendencia uniforme con cierta variación con respecto de la línea de tendencia, para el caso de la tasa de depreciación del tipo de cambio, Brasil muestra una tendencia diferente a los otros países e igualmente se percibe mayor volatilidad de los datos.
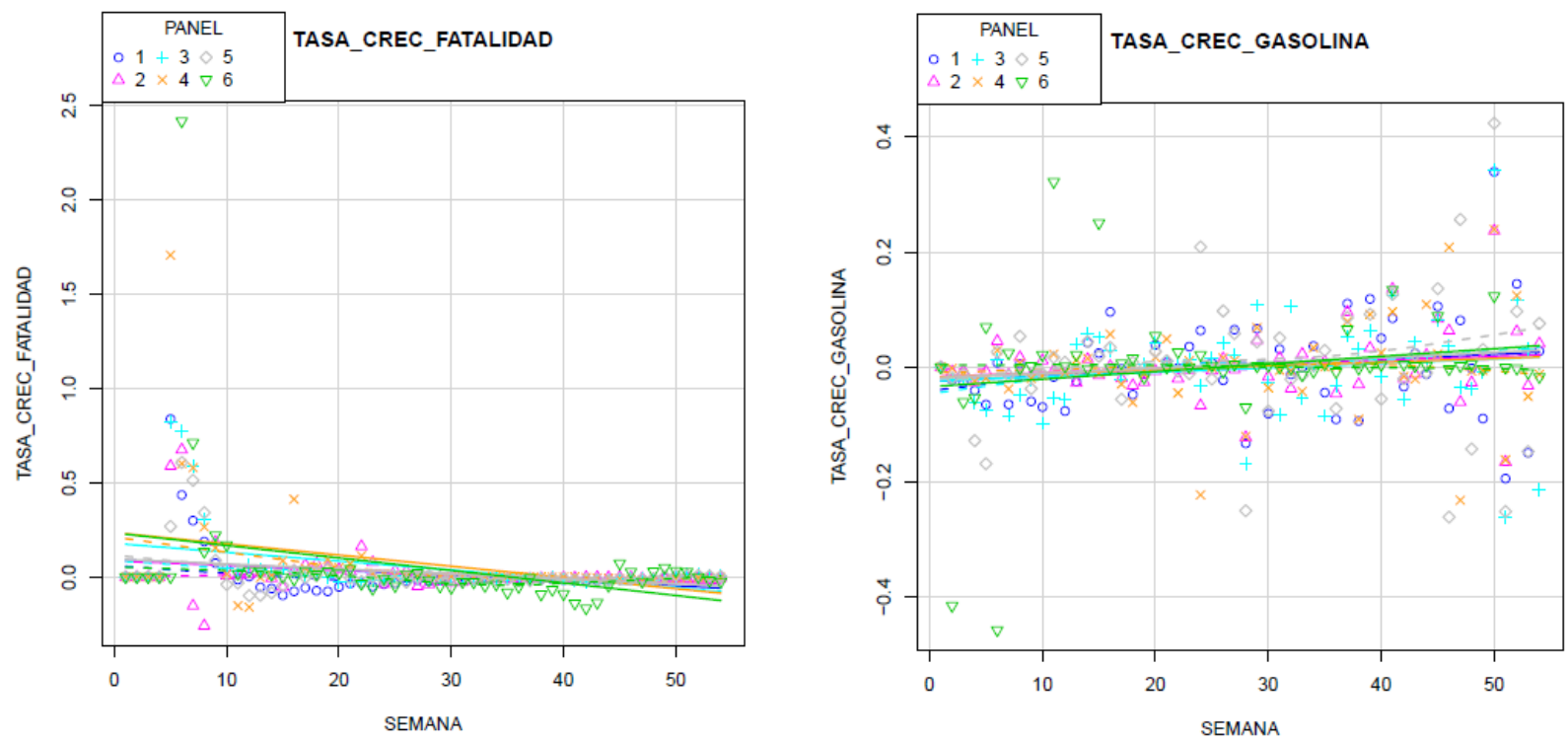

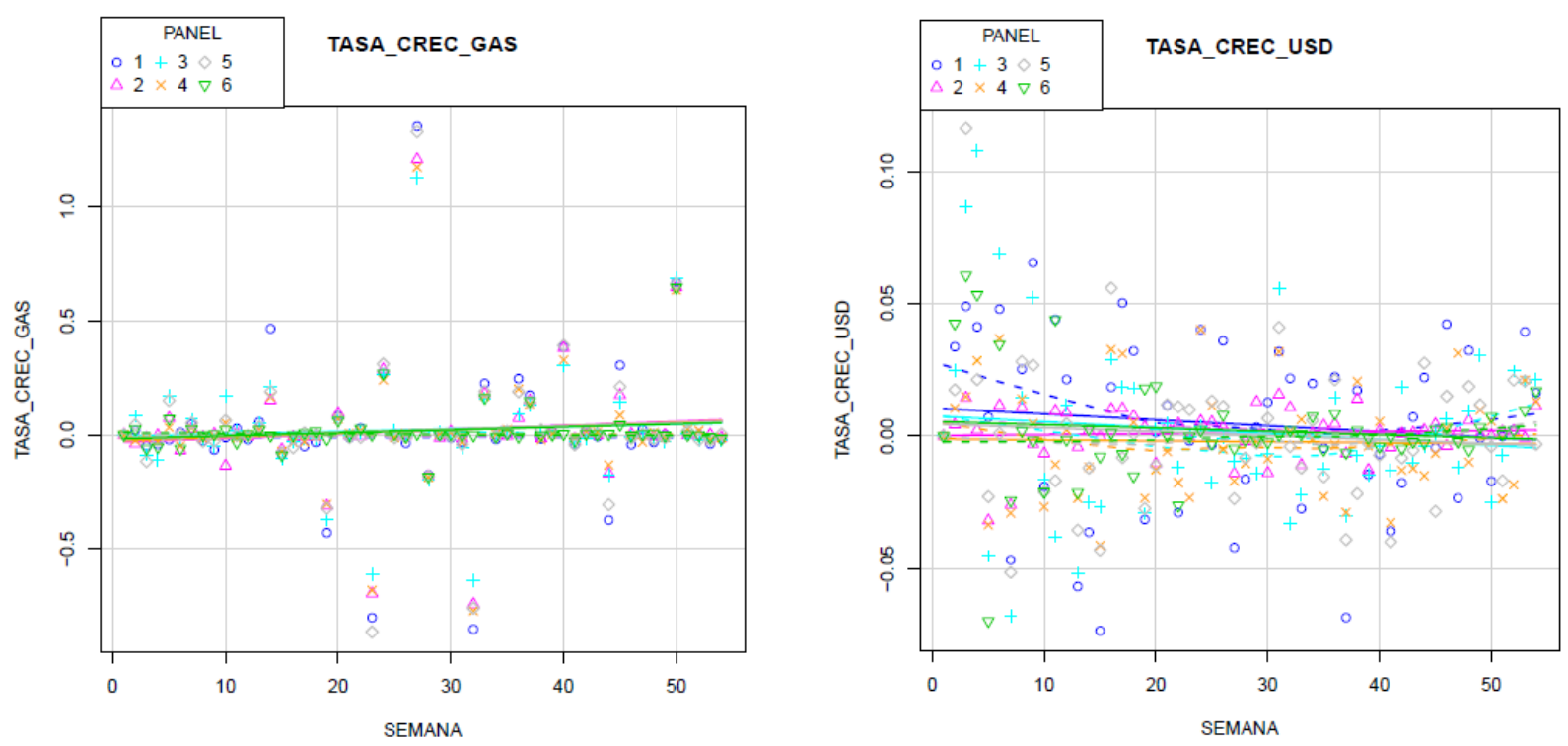

Gráfica 8. Efectos temporales y tendencias lineales Fuente: Elaboración propia con R.project

Nota. La muestra comprende 54 semanas iniciando el 26 de febrero de 2020 y terminando el 28 de febrero de 2021.

En la Tabla 3 se presenta la prueba de estacionariedad Dickey-Fuller Aumentada (Dickey y Fuller, 1979) para cada variable de análisis y por cada país. De acuerdo con dicha Tabla se tiene que todos los datos dentro del panel son estacionarios.

Tabla 3. Prueba de Dickey-Fuller Aumentada de estacionariedad de variables por país.

\begin{tabular}{|c|l|l|l|l|}
\hline Variable & \multicolumn{1}{|c|}{ País } & Estimador & P-value & \multicolumn{1}{c|}{ Observaciones } \\
\hline \multirow{4}{*}{$\begin{array}{c}\text { Tasa de } \\
\text { crecimiento } \\
\text { precios de } \\
\text { gasolina }\end{array}$} & Brasil & -13.931 & 0.01 & Estacionaria en niveles \\
\cline { 2 - 5 } & Perú & -10.924 & 0.01 & Estacionaria en niveles \\
\cline { 2 - 5 } & México & -10.48 & 0.01 & Estacionaria en niveles \\
\cline { 2 - 5 } & Chile & -11.262 & 0.01 & Estacionaria en niveles \\
\cline { 2 - 5 } & Colombia & -12.526 & 0.01 & Estacionaria en niveles \\
\cline { 2 - 5 } & Uruguay & -6.8914 & 0.01 & Estacionaria en niveles \\
\hline \multirow{4}{*}{$\begin{array}{c}\text { Tasa de } \\
\text { crecimiento } \\
\text { precios del } \\
\text { gas natural }\end{array}$} & Brasil & -2.5667 & 0.3463 & No estacionaria en niveles \\
\cline { 2 - 5 } & & -8.2508 & 0.01 & Estacionaria en diferencias \\
\cline { 2 - 5 } & Perú & -2.0204 & 0.5663 & No estacionaria en niveles \\
\cline { 2 - 5 } & & -8.3018 & 0.01 & Estacionaria en diferencias \\
\cline { 2 - 5 } & & -2.4239 & 0.4038 & No estacionaria en niveles \\
& & -8.2156 & 0.01 & Estacionaria en diferencias \\
\cline { 2 - 5 } & Colombia & -2.0978 & 0.5351 & No estacionaria en niveles \\
& & -8.0527 & 0.01 & Estacionaria en diferencias \\
\hline
\end{tabular}


REMEF (The Mexican Journal of Economics and Finance)

Impacto de la pandemia COVID-19 en los precios de la gasolina y el gas natural en las principales economías de Latinoamérica

\begin{tabular}{|c|c|c|c|c|}
\hline & Uruguay & $\begin{array}{l}-1.5633 \\
-7.6191\end{array}$ & $\begin{array}{l}0.7504 \\
0.01\end{array}$ & $\begin{array}{l}\text { No estacionaria en niveles } \\
\text { Estacionaria en diferencias }\end{array}$ \\
\hline \multirow{6}{*}{$\begin{array}{c}\text { Tasa de } \\
\text { depreciación } \\
\text { del tipo de } \\
\text { cambio a USD }\end{array}$} & Brasil & -3.7302 & 0.03049 & Estacionaria en niveles \\
\hline & Perú & -3.4961 & 0.05015 & Estacionaria en niveles \\
\hline & México & -4.5558 & 0.01 & Estacionaria en niveles \\
\hline & Chile & -3.8235 & 0.0238 & Estacionaria en niveles \\
\hline & Colombia & -3.8921 & 0.02085 & Estacionaria en niveles \\
\hline & Uruguay & -6.6991 & 0.01 & Estacionaria en niveles \\
\hline \multirow{6}{*}{$\begin{array}{c}\text { Tasa de } \\
\text { crecimiento } \\
\text { del Índice de } \\
\text { fatalidad }\end{array}$} & Brasil & -4.006 & 0.01593 & Estacionaria en niveles \\
\hline & Perú & -5.5239 & 0.01 & Estacionaria en niveles \\
\hline & México & -3.1473 & 0.1124 & No estacionaria en niveles \\
\hline & Chile & -5.3463 & 0.01 & Estacionaria en niveles \\
\hline & Colombia & -2.5295 & 0.3613 & No estacionaria en niveles \\
\hline & Uruguay & -5.7323 & 0.01 & Estacionaria en niveles \\
\hline
\end{tabular}

La Gráfica 9 muestra el comportamiento de las medias de las tasas de crecimiento de las variables en el corte transversal de los 6 países de estudio. Como se puede apreciar, los efectos de corte transversal son evidentes en todas las variables.
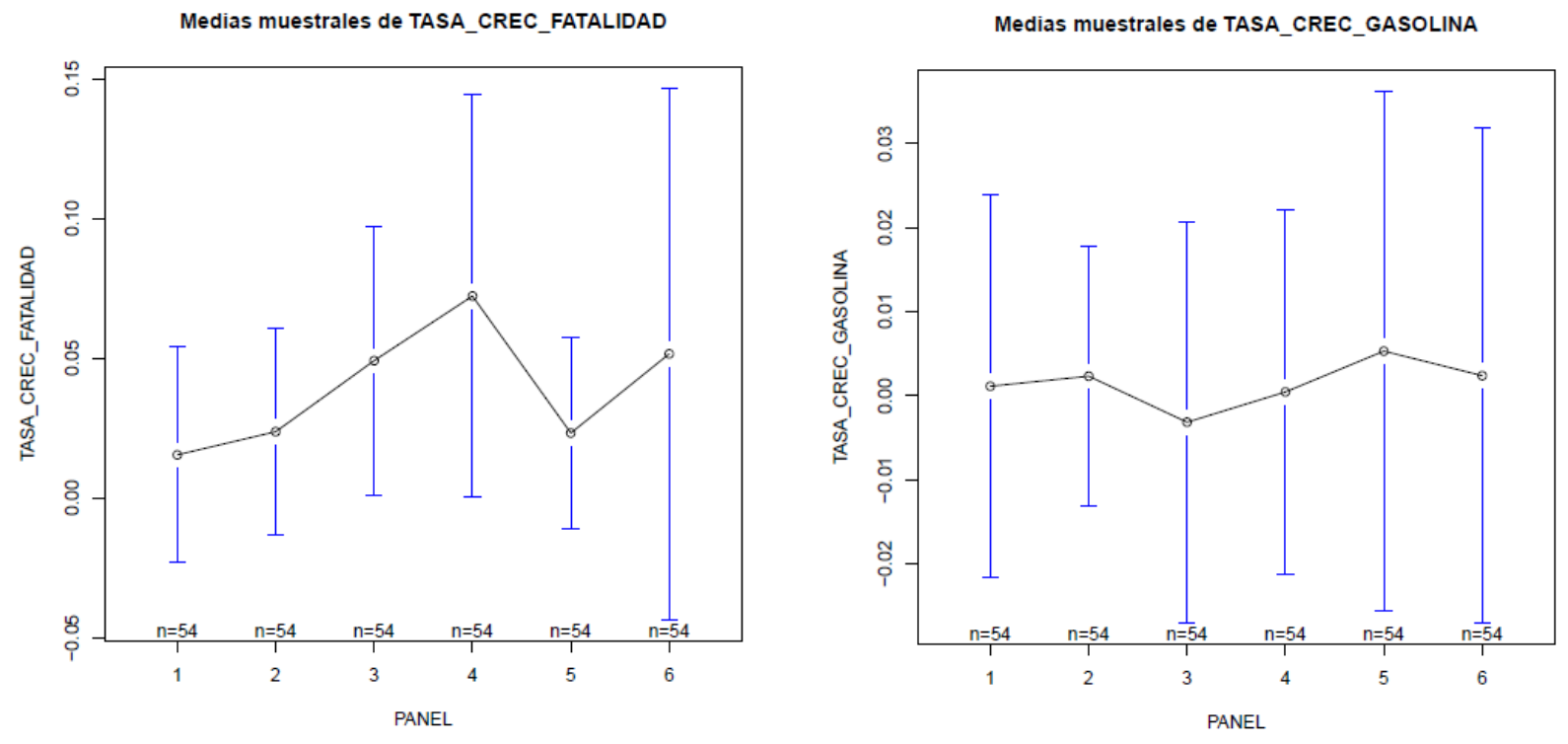

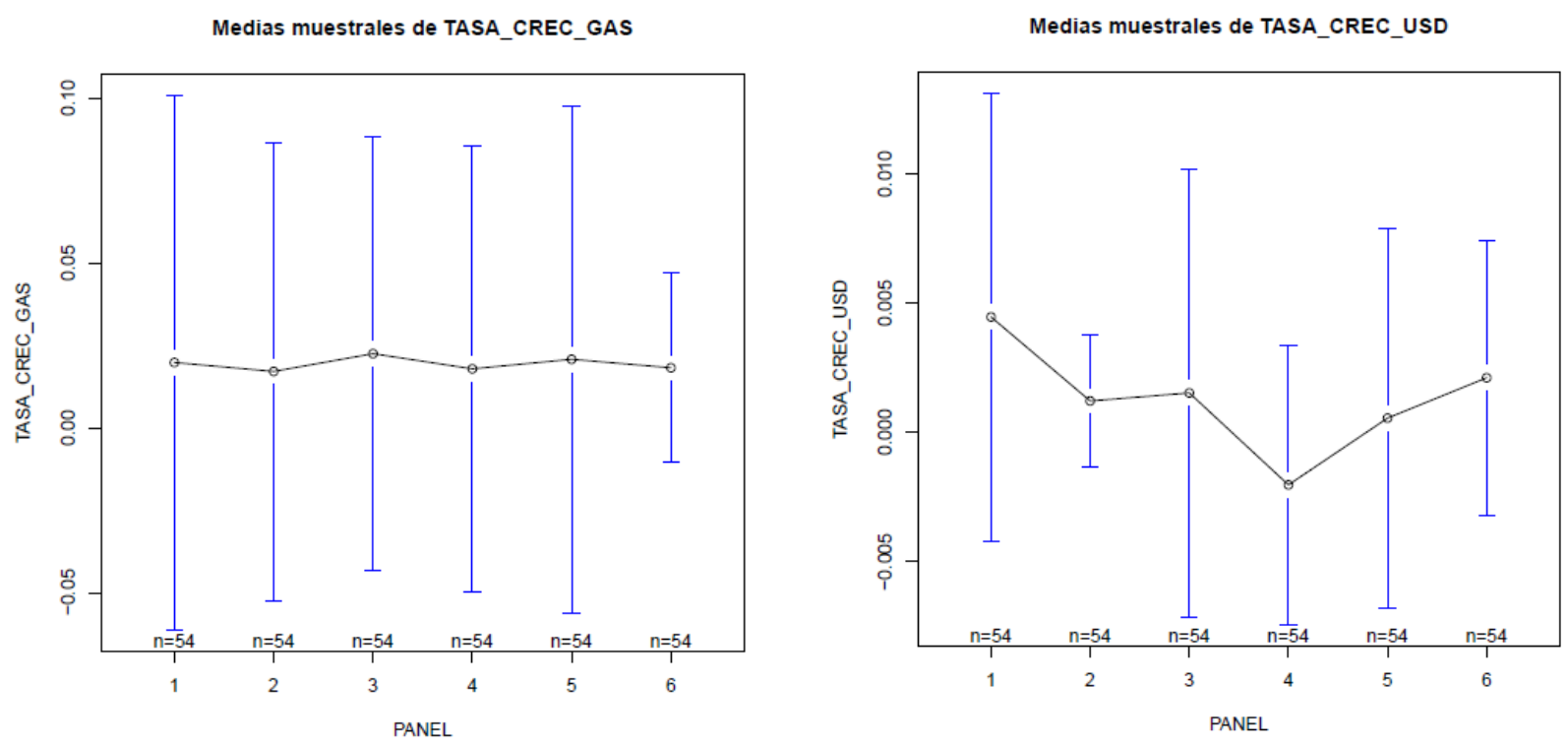

Gráfica 9. Efectos de corte transversal

Fuente: Elaboración propias con R.project

Nota. Los países representados por paneles son: 1 Brasil, 2 Perú, 3 México, 4 Chile, 5 Colombia y 6 Uruguay.

A continuación, se aplica una prueba de estacionariedad en panel de manera individual de Hadri (2000) para identificar los interceptos bajo la hipótesis donde no existe raíz unitaria en todas las variables de estudio. El resultado se muestra en el Tabla 4 en donde se considera la hipótesis alternativa de que al menos una serie tiene raíz unitaria.

Tabla 4. Prueba de Hadri (2000)

\begin{tabular}{|c|c|c|c|c|}
\hline & $\begin{array}{c}\text { Tasa de } \\
\text { crecimiento } \\
\text { del precio de } \\
\text { la gasolina }\end{array}$ & $\begin{array}{c}\text { Tasa de } \\
\text { crecimiento } \\
\text { del precio del } \\
\text { gas }\end{array}$ & $\begin{array}{c}\text { Tasa de } \\
\text { depreciación } \\
\text { del tipo de } \\
\text { cambio a USD }\end{array}$ & $\begin{array}{c}\text { Tasa de } \\
\text { crecimiento } \\
\text { del índice de } \\
\text { fatalidad }\end{array}$ \\
\hline$p$-value & 0.1178 & $<2.2 \mathrm{e}-16$ & $<2.2 \mathrm{e}-16$ & $<2.2 \mathrm{e}-16$ \\
\hline$Z$ & 1.1862 & 39.374 & 40.745 & 9.4898 \\
\hline$H_{a}:$ & Rechaza & \multicolumn{2}{|c|}{ Al menos una serie presenta raíz unitaria } \\
\hline
\end{tabular}

Fuente: Elaboración propia con R.project

También se realiza la prueba de raíz unitaria Maddala-Wu (1999) en donde se indaga sobre la heterogeneidad entre los países en el panel. La prueba considera la hipótesis alternativa en donde los datos son estacionarios y es mostrada en la Tabla 5. 
Tabla 5. Prueba de raíz unitaria Maddala-Wu (1999)

\begin{tabular}{|c|c|c|c|c|}
\hline & $\begin{array}{c}\text { Tasa de } \\
\text { crecimiento } \\
\text { del precio de } \\
\text { la gasolina }\end{array}$ & $\begin{array}{c}\text { Tasa de } \\
\text { crecimiento } \\
\text { del precio del } \\
\text { gas }\end{array}$ & $\begin{array}{c}\text { Tasa de } \\
\text { depreciación } \\
\text { del tipo de } \\
\text { cambio a USD }\end{array}$ & $\begin{array}{c}\text { Tasa de } \\
\text { crecimiento } \\
\text { del índice de } \\
\text { fatalidad }\end{array}$ \\
\hline $\mathrm{p}$-value & $<2.2 \mathrm{e}-16$ & 0.9863 & $2.118 \mathrm{e}-07$ & $3.207 \mathrm{e}-14$ \\
\hline$\chi^{2}$ & 510.28 & 3.8297 & 54.619 & 90.967 \\
\hline$H a:$ & Estacionaria & No estacionaria & Estacionaria & Estacionaria \\
\hline
\end{tabular}

Fuente: Elaboración propia con R.project

Con base en los resultados de las pruebas anteriores se busca ahora identificar la especificación de los modelos de efectos fijos y efectos aleatorios, ambos a 2 vías.

\section{Discusión de los resultados empíricos}

En las Tablas 6 y 7 sólo se muestran las variables que resultaron significativas para cada uno de los paneles de análisis; el resto de las variables tuvieron $p$-values mayores a 0.5 . Se destaca, primero, que el modelo de efectos aleatorios tiene mayor significancia. Además, de acuerdo con la información que nos arrojan el modelo y las gráficas es factible suponer que para cada unidad transversal existe un intercepto diferente, que económicamente supone, que existe un perfil individual para cada país.

Tabla 6. Modelos de datos panel para la variable dependiente Tasa de Crecimiento de los Precios de la gasolina

\begin{tabular}{|c|c|c|c|c|}
\hline Coeficiente & Estimado & Error Estándar & p-value \\
\hline \multicolumn{5}{|c|}{ Efectos fijos 2 vías } \\
\hline TC_FATALIDAD & -0.12728 & 0.02815 & $9.29 \mathrm{e}-06$ & $* * *$ \\
\hline TC_USD & -0.59673 & 0.23670 & 0.01229 & $*$ \\
\hline Estadística $F$ & 14.096 & p-value & $1.5296 \mathrm{e}-06$ & \\
\hline \multicolumn{5}{|c|}{ Efectos aleatorios 2 vías } \\
\hline Intercepto & 0.0056891 & 0.0083702 & 0.049670 & $*$ \\
\hline TC_FATALIDAD & -0.112155 & 0.0240961 & $3.25 \mathrm{E}-06$ & $* * *$ \\
\hline TC_USD & -0.5753773 & 0.2090705 & 0.005922 & $* *$ \\
\hline TC_GAS & 0.0445817 & 0.0269911 & 0.098592 & $\#$ \\
\hline$\chi^{2}$ & 35.0184 & p-value & $1.2074 \mathrm{e}-07$ & \\
\hline \multicolumn{5}{|c|}{ Nota: Significancia “***”.1\%, “**”1\%, “*”5\%, “\#” $10 \%$} \\
Fuente: Elaboración propia con R.project \\
\hline
\end{tabular}

En la Tabla 6 sólo se muestran las variables que resultan significativas para cada uno de los paneles de análisis. En el modelo de efectos aleatorios se puede observar que el crecimiento en los 
precios de la gasolina está explicado, primero, por un intercepto individual para cada país y, después, por la tasa de fatalidad por COVID-19 de manera inversa. Esto significa que si incrementa la tasa de fatalidad, entonces disminuye la tasa de crecimiento de los precios del gas. Esto último se debe, en gran medida, a los siguientes factores: 1) que los precios del petróleo, al igual que el precio de la gasolina, se valúan diariamente, mientras que los precios del gas natural son valuados con base en el precio spot Henry Hub que se calcula con periodicidad mensual; 2) la demanda del gasolina en los hogares y en la industria tuvo una disminución significativa durante el periodo de mayor confinamiento social; y 3) para evitar los altos costos de almacenamiento se tuvo que disminuir el precio de la gasolina. Es también razonable encontrar también, en el análisis panel, que el crecimiento del gas natural está asociado al crecimiento de la gasolina de manera positiva, aunque el efecto no es muy fuerte. Esto se debe a que la relación fluye de manera más fuerte de la gasolina hacia el gas y no de manera inversa por la comercialización y sus derivados. Por último, es importante mencionar, de acuerdo al análisis panel realizado que si el tipo de cambio crece, entonces el precio de la gasolina decrece pero en mayor proporción.

En la Tabla 7 se observan los siguientes dos comportamientos que provienen de los modelos. El primero de efectos fijos a 2 vías muestra que la tasa de crecimiento del índice de fatalidad afecta negativamente la tasa de crecimiento del precio de la gasolina. El segundo de efectos aleatorios a 2 vías indica que la tasa de crecimiento del índice de fatalidad no tiene efectos sobre la tasa de crecimiento de los precios del gas. El modelo de efectos aleatorios es preferible al modelo de efectos fijos ya que tiene un nivel de significancia mayor y al igual que el modelo anterior, es factible suponer un intercepto individual para cada país.

Tabla 7. Modelos de datos panel para la variable dependiente la Tasa de Crecimiento del Precio de gas natural

\begin{tabular}{|c|c|c|c|c|}
\hline Coeficiente & Estimado & Error Estándar & \multicolumn{2}{|c|}{$p$-value } \\
\hline \multicolumn{5}{|c|}{ Efectos fijos 2 vías } \\
\hline TC_USD & -1.09574 & 0.36817 & 0.003189 & $* * *$ \\
\hline Estadística $F$ & 8.85781 & $p$-value & 0.0031891 & $* * *$ \\
\hline \multicolumn{5}{|c|}{ Efectos aleatorios 2 vías } \\
\hline Intercepto & 0.020868 & 0.030169 & 0.048912 & $*$ \\
\hline TC_USD & -1.113506 & 0.354492 & 0.001683 & ** \\
\hline$\chi^{2}$ & 9.86669 & $p$-value & 0.001683 & $* *$ \\
\hline
\end{tabular}

En el modelo de efectos aleatorios la tasa de fatalidad, TC_FATALIDAD, no tiene efectos significativos en la tasa de crecimiento del precio del gas ya que su $p$-value se encuentra cercano a 0.5. Esto se debe, en gran medida, a los siguientes factores: 1) que los precios del gas natural en la mayoría de las economías de la muestra son valuados con base en el precio spot Henry Hub de manera mensual a diferencia del precio del petróleo que se valúa diariamente en los mercados internacionales, y 2) la demanda del gas natural en los hogares tuvo un aumento significativo durante el periodo de mayor confinamiento en la pandemia y, al mismo tiempo, los costos de almacenamiento 
se mantuvieron bajos. No obstante, se observa que únicamente la tasa de depreciación del tipo cambio impacta al crecimiento de los precios de gas natural de manera negativa. Es decir, un incremento en el crecimiento del tipo de cambio recae en un decrecimiento en el precio de gas natural. En este caso se tienen precios más estables, incluso en el periodo de pandemia, a diferencia de la gasolina que sí muestra efectos por los efectos de la crisis sanitaria.

\section{Conclusiones}

La pandemia COVID-19 es un fenómeno global que afecta a los precios de los energéticos de las economías bajo estudio, los efectos en las tasas de crecimiento de los índices de fatalidad de los países en la muestra únicamente tienen efectos negativos sobre los precios de gasolina en el transcurso del primer año de la pandemia. Además, existieron factores económicos que impactaron los precios de la gasolina a nivel global. Los precios fueron arrastrados hacia la baja por el confinamiento y las fuerzas de mercado, como se explicado detalladamente en la sección 5.

Los resultados empíricos revelan que los movimientos entre el precio de la gasolina y las variables independientes, tasa de depreciación del tipo de cambio e índice de fatalidad por COVID19, fueron significativos y negativamente relacionados durante el primer año de la pandemia. Además, el crecimiento en los precios de gas natural es significativo y positivo. Para las economías analizadas, los resultados son congruentes con el enfoque de riqueza y el modelo internacional de asignación de carteras (Golub, 1983; y Krugman, 1983), pues en la muestra existen países productores de petróleo, México, Brasil, Chile, Colombia y Perú, únicamente Uruguay sale de la asignación productora y es claramente visible en las gráficas y pruebas.

El modelo de efectos aleatorios desarrollado en la presente investigación muestra evidencia empírica de que la tasa de fatalidad no tiene efectos significativos en la tasa de crecimiento del precio del gas. Esto se debe, en gran medida, a la contribución del confinamiento en las fuerzas del mercado, como se ha expuesto en la sección 5. No obstante, la relación negativa entre las variables de crecimiento entre el precio del gas natural y tipo de cambio pueden explicarse también por el contagio en los mercados financieros durante los episodios de crisis, como se sugiere (Živkov et al. 2019).

Un año de análisis de la pandemia brinda una perspectiva más general de las consecuencias y decisiones que se deben afrontar en el futuro, las relaciones expuestas permiten a los tomadores de decisiones en políticas públicas tomar acciones frente a los movimientos de los tipos de cambio y los precios de la gasolina y el gas natural ya que estos afectan a los costos de producción y rentabilidad afectando negativamente la tasa de desempleo ( $c f$. CEPAL, 2021).

Además, este análisis contribuye a generar y reforzar propuestas en materia de políticas públicas de salud. Las recomendaciones en esta materia deberían estar encaminadas hacia la disminución de la letalidad por COVID-19 a través de acciones específicas de salud (pruebas clínicas, seguimiento y rastreo de posibles casos de infectados, uso de cubre bocas, sistemas nacionales de vacunación y otras medidas sobre el comportamiento social), ya que esto podría reducir la volatilidad que genera el índice de fatalidad en los precios de la gasolina. Así, con precios más estables en la gasolina se tendría un efecto positivo en diversos sectores productivos y en las unidades familiares. 
Reinhart (2020) además comenta "este es un momento que toma todo para las políticas fiscales y monetarias a gran escala y fuera de la caja”, y en este sentido la contribución del análisis expuesto en esta investigación refuerza lo dicho sobre las políticas monetarias, que influyen directamente a los precios de los energéticos y de ahí se desencadenan efectos hasta las familias.

Por último, en la agenda de investigación futura se pretende utilizar modelos de paneles dinámicos con estimación mediante el método de momentos generalizados (MMG) y considerando una mayor horizonte de tiempo.

\section{Referencias}

[1] Aali-Bujari, A., Venegas-Martínez, F., and Palafox-Roca, A. O. (2017). Impact of Energy Consumption on Economic Growth in Major OECD Economies (1977-2014): A Panel Data Approach. International Journal of Energy Economics and Policy, 7(2), 1-8. Obtenido de https://www.econjournals.com/index.php/ijeep/article/view/4146/2615

[2] Abadie, L. M. (2020). Current expectations and actual values for the clean spark spread: The case of Spain in the Covid-19 crisis. Journal of Cleaner Production. doi:https://doi.org/10.1016/j.jclepro.2020.124842

[3] Abadie, L. M., Chamorro, J. M., Huclin, S., and van de Ven, D. J. (2020). On flexible hydropower and security of supply: Spain beyond 2020. Energy. 203, 15 July 2020, 117869 doi:https://doi.org/10.1016/j.energy.2020.117869

[4] Amankwah-Amoah, J. (2020). Stepping up and stepping out of COVID-19: New challenges for environmental sustainability policies in the global airline industry. Journal of Cleaner Production, 271. doi:https://doi.org/10.1016/j.jclepro.2020.123000

[5] Arellano, M., y Bover, O. (1990). La econometría de datos de panel. Investigaciones económicas, 14(1), 3-45.

[6] Barbier, E. B., and Burgess, J. C. (2020). Sustainability and development after COVID-19. World Development, 135. doi:10.1016/j.worlddev.2020.105082

[7] Barbosa, F., Bresciani, G., Graham, P., Nyquist, S., and Yanosek, K. (2020). Oil and gas after COVID-19: The day of reckoning or a new age of opportunity. McKinsey \& Company.

[8] Bolton, P., Després, M., Silva, L. A., Samama, F., and Svartzman, R. (2020). The green swan. BIS.

[9] Carvajal, O. H. y Bermúdez, L. E. (2020). Coyuntura del coronavirus covid-19 en países medianos productores de petróleo $i$ Qué hacer en el caso de Colombia? Boletim de Conjuntura (BOCA), 2(5), 6371. doi:http://doi.org/10.5281/zenodo.3764261

[10] Clavellina-Miller, J. L. y Domínguez-Rivas, M. I. (2020). Implicaciones económicas de la pandemia por COVID-19 y opciones de política. México: Notas estratégicas: Dirección General de Finanzas, Senado de la República.

[11] Dickey, D. A., and Fuller, W. A. (1979). Distribution of the Estimators for Autoregressive Time Series with a Unit Root. Journal of the American Statistical Association, 74, 427-431.

[12] Escribano, G. y Lázaro, L. (2020). Energía, clima y coronavirus. Madrid, España.: Real Instituto Elcano. Obtenido de https://www.researchgate.net/profile/Gonzalo-Escribano2/publication/347003097_Energia_clima_y_coronavirus/links/5fd77efc45851553a0b5afd5/Energia -clima-y-coronavirus.pdf

[13] Fondo Monetario Internacional. (24 de 02 de 2020). Recession: When Bad Times Prevail. Obtenido de International Monetary Fund: https://www.imf.org/external/pubs/ft/fandd/basics/recess.htm

[14] Galindo, L. M., y Reyes, O. (2015). Meta-análisis de las elasticidades ingreso y precio de la demanda de gasolina: implicaciones de política pública para América Latina. Revista CEPAL.117, 7-25. 
REMEF (The Mexican Journal of Economics and Finance)

Impacto de la pandemia COVID-19 en los precios de la gasolina y el gas natural

en las principales economías de Latinoamérica

[15] García D. P. (2020). Las importaciones de gas natural licuado (GNL) a España y al conjunto de la Unión Europea desde Estados Unidos: del debate sobre el fraking y la geopolitica de los flujos de suministros energéticos. Finisterra - Revista Portuguesa de Geografía. 55(113), 135-155. doi:10.18055/Finis16614

[16] Golub, S. S. (1983). Oil Prices and Exchange Rates. The Economic Journal, 93(371), 576-593. doi:https://doi.org/10.2307/223239

[17] Gómez, J. E., Hirs, J., and Uribe, J. M. (2020). Giving and Receiving:Exploring the Predictive Causality Between Oil Prices and Exchange Rates. International Finance, 23(1), 175-194. doi:https://doi.org/10.1111/infi.12354

[18] Hadri, K. (2000). Testing for Stationarity in Heterogeneous Panel Data. Econometric Journal, 3(2), 148161.

[19] Kim, J.-M., and Jung, H. (2018). Relationship Between Oil Price and Exchange Rate by FDA and Copula. Applied Economics, 50(22), 2486-2499. doi:https://doi.org/10.1080/00036846.2017.1400652

[20] Krugman, P. (1983). Oil Shocks and Exchange Rate Dynamics. Exchange Rates and International Macroeconomics, 259-284.

[21] López-Herrera, F. y Rodríguez-Benavides, D. (2020). Efectos asimétricos en la relación de los precios bursátiles con el tipo de cambio, los precios mundiales del petróleo y la tasa de interés ante la pandemia de Covid-19. Panorama Económico, 16(32), 169-181. Obtenido de http://www.panoramaeconomico.mx/ojs/index.php/PE/article/view/14

[22] Maddala, G., and Wu, S. (1999). A Comparative Study of Unit Root Test with Panel Data and a New Simple Test. Oxford Bulletin of Economics y Statistics, 61, 631-652.

[23] Meher, B. K., Hawaldar, I. T., Mohapatra, L., and Sarea, A. (2020). The Impact of COVID-19 on Price Volatility of Crude Oil and Natural Gas Listed on Multi Commodity Exchange of India. International Journal of Energy Economics and Policy, 10(5), 422-431. doi:https://doi.org/10.32479/ijeep.10047

[24] Mendoza-Rivera, R. J., Lozano-Díez, J. A., y Venegas-Martínez, F. (2020). Impacto de la pandemia Covid19 en variables financieras relevantes en las principales economías de Latinoamérica. Economía Teoría $\begin{array}{llll}Y & \text { Práctica } & \text { (Número } & \text { Especial }\end{array}$ doi:http://dx.doi.org/10.24275/ETYPUAM/NE/E052020/Mendoza

[25] Reinhart, C. (2020). This time truly is different. Cambrige, USA: Project Syndicate. Obtenido de https://www.advisorperspectives.com/commentaries/2020/03/23/this-time-truly-is-different.pdf

[26] Stiglitz, J. (2017). Where Macroeconomics Went Wrong. NBER. Working Paper Series.

[27] Summers, L. H. (2000). International financial crises: causes, prevention and cures. American Economic Review, 90(2), 1-16. doi:10.1257/aer.90.2.1

[28] Thenmozhi, M., and Srinivasan, N. (2016). Co-movement of Oil Price, Exchange Rate and Stock Index of Major Oil Importing Countries: A Wavelet Coherence Approach. The Journal of Developing Areas, 50(5), 85-102. doi:https://doi.org/10.1353/jda.2016.0036

[29] Villarreal-Samaniego, D. (2021). The dynamics of oil prices, COVID-19, and exchange rates in five emerging economies in the atypical first quarter of 2020. Estudios Gerenciales, 37(158), 17-27. doi:https://doi.org/10.18046/j.estger.2021.158.4042

[30] Wu, C. C., Chung, H., and Chang, Y. H. (2012). The Economic Value of Comovement Between Oil Price and Exchange Rate Using Copula-Based GARCH Models. Energy Economics, 34(1), $270-282$. doi:https://doi.org/10.1016/j.eneco.2011.07.007

[31] Živkov, D., Njegić, J., and Balaban, S. (2019). Revealing the Nexus Between Oil and Exchange Rate in the Major Emerging Markets - The Timescale Analysis. International Journal of Finance and Economics, 24(2), 685-697. doi:https://doi.org/10.1002/ijfe.1686 